\title{
Industry 4.0 and the Civil Construction in Brazil
}

\section{SIGRADI2018 TECHNOPOLITICAS \\ xxii congresso da sociedade iberoamericana de gráfica digital 22 th conference of the iberoamerican society of digital graphics $07|08| 09$ |novembro|2018 iau usp | são carlos | sp br}

\author{
Elza Luli Miyasaka \\ Dep. Arq e Urb (DAU) - Viçosa Federal University | Brazil | mel@ufv.br \\ Márcio Minto Fabricio \\ IAU-USP | Brazil | marcio@sc.usp.br \\ Ingrid Paoletti \\ ABC Dep - POLIMI | Italy | ingrid.paoletti@polimi.it
}

\begin{abstract}
This paper aims to make an exploratory research about the dissemination of the purpose of Industry 4.0 concept in Brazil, that was introduced by Germany in 2011, especially in Architecture, Engineering and Construction sector. For this aim it was made a survey in magazines on line. As results was verified that the area that is discussing the concept are essentially productions engineering with a inexpressive repercussion in civil construction.
\end{abstract}

Keywords: Industry 4.0, Digital Fabrication, Technopolítics, Brazil.

\section{INTRODUÇÃO}

O processo de digitalização ou de numerização dos sistemas analógicos para uma codificação em matrizes organizadas por 0 (zero) e 1 (um) ainda é a base para a lógica que transformou toda a racionalidade do sistema digital e que deu o salto na tecnologia, modificando os paradigmas nos vários setores da sociedade (BRETON; PROUX, 2006; MATTELART, 2002). O computador é a "máquina universal" utilizada em todas as áreas e disciplinas; desempenha tarefas mentais, realiza mudanças nos processos e nas organizações. Essas mudanças se retroalimentam, ampliando ainda mais as possibilidades oferecidas (BRYNJOLFSSON; McAFEE, 2011).

No contexto atual, é possível utilizar a tecnologia de sensores aplicados em locais estratégicos, que verificam a umidade do ambiente e decidem quanto e como irrigar plantações em diversas regiões; sistemas urbanos que podem ser organizados a partir de dispositivos que informam quando e onde coletar lixos, abastecimentos de energia, água e esgotos, monitoramento de trânsito etc. Microprocessadores incorporados a objetos e máquinas possibilitam a sua programação para que se tornem autossustentáveis e inteligentes, máquinas recebem pedidos e podem confeccionar o produto, tais como medicamentos ou vestiários, e enviar diretamente para a entrega ao cliente; a internet possibilita o compartilhamento de trabalhos de qualquer ponto geográfico em tempo real.

"Automatizar" é entendido como tornar-se independente de algum processo. A palavra foi diminuída para automação, que vem do grego automotos, de movimento, e ion, do latim, que significa estado; é a substituição do trabalho físico e mental do homem pelas máquinas (KATSUNDO, 1994). A automação, pelo dicionário Britânica, significa a aplicação de máquinas em tarefas executadas por seres humanos. Atualmente, é aplicada para um conjunto de técnicas com a finalidade de tornar automática a realização de tarefas, substituindo a força humana, física e mental, por dispositivos eletromecânicos computacionais (SILVEIRA; LIMA, 2003).

A vontade de substituir o trabalho humano pelas máquinas sempre esteve presente, especialmente quando associada ao aumento da produtividade e à diminuição dos custos. As mais antigas máquinasferramenta foram as mandriladoras de canhão dos séculos 16, 17 e 18. São máquinas flexíveis que permitem a adaptação de diferentes ferramentas, utilizadas para usinagem, perfurações, fresagem, mandrilagem e torneamento, sem a necessidade da retirada do objeto. Este equipamento era movido por um eixo ligado a uma roda d'água externa, que movimentava os trilhos, polias e cordas (MORAES; ABREU, 2006).

De acordo com a história, os processos de mecanização e automatização passaram por diversas etapas, caracterizadas hora pelo seu fornecimento de energia, hora pela sua organização na forma de produzir. No contexto atual, verifica-se um processo que é caracterizado pelo uso extensivo dos processos digitalizados e se configura para uma nova proposta de atenção ao mercado consumidor. Essa conformação é também conhecida como Indústria 4.0, pois propõe uma reorganização da produção e 4.0 porque se encontra na 4ํ. Revolução Industrial.

A primeira versão da Indústria 4.0 (14.0) foi criada na feira de Hannover (2011), para anunciar uma estratégia do governo alemão para o desenvolvimento de alta tecnologia que promove a digitalização da indústria "Estratégia High-tech 2020" (ANTONIO, 2016). Os autores consideram que o movimento é uma modificação dos meios de produção. 
A Indústria 4.0 descreve um projeto de sistemas inteligentes e autônomos de fábricas e máquinas robóticas, com uso da nanotecnologia e produção aditiva, conectados em rede e com o consumidor. Os processos de produção são descentralizados nos locais de consumo, com dispositivos para avaliar problemas de desempenho, disseminando o conhecimento, e não somente o produto (LEE; KAO; YANG, 2014) (LASI et al., 2014) (LARIZZA, 2016) (TRENTESAUX, BORANGIU; THOMAS, 2016) (SOMMER, 2015) (SHAFIQA et al., 2015) (WANG et al., 2015).

A Indústria 4.0 tem como alvos principais: 1. Produzir de forma customizada, atendendo cada vez mais às necessidades de personalização dos objetos de acordo com a demanda; 2. Adaptar a manufatura de forma flexível, para atender à cadeia de produção; 3 . Reconhecer de forma inteligente informações a respeito de objetos e máquinas em rede; 4. Apropriação da interação com as máquinas contemporâneas como robôs e máquinas autônomas e 5. Adequação dos serviços oferecidos que contribuam na cadeia de valor (SHAFIQA et al., 2015).

A iniciativa da Alemanha Industry 4.0 (I4.0) - Indústria 4.0 -, em 2011, impulsionou os territórios Europeus às oportunidades tecnológicas, aos desafios científicos relacionados com a entrada de novas gerações de tecnologias, de comunicação e serviços nos sistemas de produção industrial. Nos Estados Unidos, a Internet Industrial foi introduzida em 2012 pela General Eletric e foi considerada a terceira onda de inovação depois da revolução industrial (SILVEIRA; LIMA, 2003; POSADA et al., 2015).

Como referem os autores, para a obtenção da personalização em massa ou Customização Avançada (NABONI; PAOLETTI, 2015), há a necessidade da diminuição das hierarquias e de descentralização dos comandos. Um sistema de logística inteligente para manufaturar o produto em diferentes partes e algoritmos potentes que permita administrar os fluxos de informações internas e externas (ANTONIO, 2016).

Essa configuração propicia às indústrias um alto grau de autonomia, em que componentes adquirem comportamentos independentes, processam informação, aprendendo com isso, e reagem em tempo real para o autocontrole e a organização. A rede é aberta para firmar novos parceiros e criar novos modelos de acordo com os custos e riscos (ABRAMOVICI; GÖBEL; NEGES, 2015).

Dentre os principais instrumentos para essa constelação de relações entre consumidores, empresas, equipamentos, tecnologias de ponta, estão os dispositivos digitalizados, a comunicação via internet habilitada, as nuvens de dados, máquinas inteligentes das fábricas com alta capacidade tecnológica e, evidentemente, os trabalhadores responsáveis pelo desenvolvimento, captura, análise, armazenamento e resposta dos dados.

\section{A CONSTRUÇÃO CIVIL}

As tecnologias empregadas na indústria da construção civil têm acompanhado gradativamente o processo de automatização e autocontrole dos mecanismos de manutenção do maquinário, gestão de metadados de clientes e manufatura do produto final tendo como finalidade reunir esforços para a melhor detecção das necessidades dos usuários e agregar competências entre as indústrias no sentido de formar uma rede complexa de produção. Diante disso, o objetivo deste artigo é verificar as discussões sobre a temática Indústria 4.0 no território brasileiro e, em especial no setor da construção civil brasileira.

Para a consolidação da automatização na construção civil, são necessários diferentes esforços (BALAGUER; ABDERRAHIM, 2008):

1. Integração entre os vários processos do desenvolvimento do edifício, com retroalimentação de dados que alimentam outros estágios, diversificação dos projetos, utilizando-se elementos similares unificados, e padronização dos programas para uma rápida alteração entre as diversas disciplinas.

2. Pré-fabricação de novos materiais que não só o concreto. Customização em massa com a utilização do conceito CIM (Computer Integrated Manufacturing), que significa 0 entrelaçamento entre a flexibilidade de um produto com a produtividade na manufatura.

3. Robôs e máquinas automatizadas: é um ponto-chave para aumentar a produtividade, para o desenvolvimento de equipamentos robotizados com menor custo e para o aumento do nível de automação das máquinas existentes.

4. Investimentos em programas internacionais, com mudança da cultura dos operadores envolvidos diretamente no processo de construção.

A temática Indústria 4.0 ainda não tem ganhado relevância nas fábricas da construção civil, ainda mais quando se fala nas possíveis redes de conexões entre clientes, produção, máquinas com retroalimentação de dados e programação especializada (OESTERREICH; TEUTEBERG, 2016). Em geral, a indústria da construção é mais conservadora. Em muitos casos, quando os novos produtos automáticos não são complementares aos antigos, são dificilmente implementados e seu uso é mínimo (BALAGUER; ABDERRAHIM, 2008).

O conceito de 14.0 para construção compreende uma multiplicidade de tecnologias interdisciplinares no sentido de permitir a digitalização, automação e integração do processo de construção em todas as fases da cadeia de valor da construção. Várias tecnologias de comunicação e informação estão em diferentes níveis de maturidade, como, por exemplo, as plataformas BIM (Building Information Modeling), que consiste em um sistema de tratamento de informações utilizadas desde a modelagem, as simulações, a gestão de custos, as soluções para pré-fabricação, análises de consumo energético, certificação, análises pós-ocupação, a gestão de informações até as nuvens de dados, que possibilitam o desenvolvimento do projeto em suas várias disciplinas, com possibilidades de compartilhamento dos processos e, também a incorporação dos dispositivos móveis como verificadores com GPS utilizados em terraplanagem, os drones, etc.

$\mathrm{Na}$ construção civil, as tecnologias de comunicação e os dispositivos avançados como tablets já são empregados em pequena escala, e aos poucos, ocupam espaços em serviços de montagem, automação do processo de fabricação e construção, mas ainda estão longe de formar uma rede inteligente de produção voltada às necessidades dos clientes, contexto este verificado principalmente nos países industrializados. 


\section{METODOLOGIA}

Para este trabalho, realiza-se a pesquisa eletrônica do tipo exploratória. Utilizou-se a base de dados de Periódicos da Capes, SIBIUSP, e Google Acadêmico. As palavras chaves utilizadas foram Indústria 4.0 (em inglês e português), Construção Civil e Brasil. Através desse filtro, obteve-se 580 artigos, que foram verificados através do título, que estivessem há menos de 10 anos de publicação e que tratassem de discussões a respeito da nova organização através da Indústria 4.0. Dentre os resultados, foram selecionados 32 artigos, que foram lidos, analisados e avaliados sobre o seu conteúdo e interesses.

\section{RESULTADOS}

De acordo com os dados pesquisados, como mostra o gráfico abaixo, verificou-se que a principal área preocupada com a nova forma de organização do mercado é a Engenharia de Produção, seguida dos setores associados ao controle automatizado. No ramo da engenharia civil e arquitetura, foram encontrados dois artigos, sendo que um deles trata da utilização da automação para o controle de trens urbanos (VLT) e o segundo faz uma análise de um estudo de caso no contexto da Indústria 4.0.

Gráfico 1: Distribuição das áreas de atuação dos autores dos artigos pesquisados. Fonte: Autor. Data: 2018.

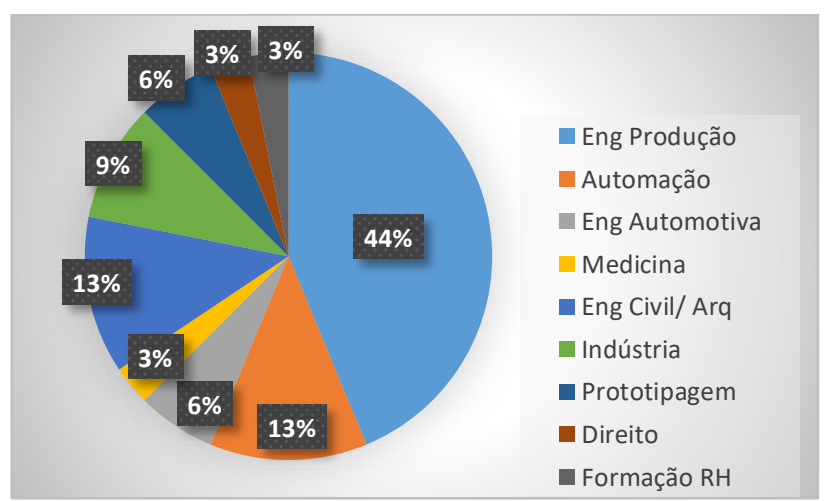

Os profissionais estão inseridos principalmente nos ramos de organização e gestão da indústria, outros com o desenvolvimento de dispositivos para controle cardíaco, regulação dos direitos e deveres e, a formação de recursos humanos para a atuação nesse novo modelo organizacional.

Em relação aos temas pesquisados, estão presentes assuntos relacionados à modularização, que em poucas palavras significa a segmentação de sistemas, produtos e processos menores, que proporciona a flexibilização da produção para a fabricação de um número maior de objetos com os recursos disponíveis e a baixos custos (CLAUDIA, 2017).

Além disso, a temática controle à distância está presente em quase $30 \%$ dos artigos, já que esse é um dos desafios colocados pela Indústria 4.0, que pretende realizar uma rede de Indústrias inteligentes, dispositivos móveis de clientes e outros bancos de dados vinculados a uma nuvem que são a base para análises minuciosas com maior capacidade de operação e elaboração de produtos, para atender às necessidades específicas dos clientes.
Como refere a literatura a respeito da atuação no território brasileiro, as pesquisas estão voltadas principalmente para a apropriação dos conhecimentos a respeito da nova proposta e, a sua adequação à produção das fábricas. É evidente que os principais setores preocupados com as inovações estratégicas estão voltados para o setor automobilístico e para os ramos que têm investimentos na área de controles automatizados, como produção de calçados, vidros, transporte urbano e empresas da área de tecnologias.

Gráfico 2: Distribuição dos assuntos apresentados nos artigos pesquisados. Fonte: Autor. Data: 2018.

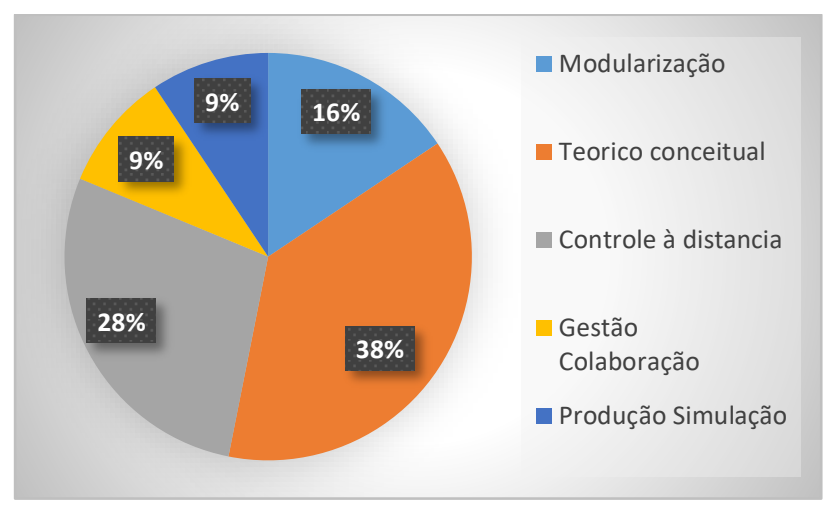

No que se refere aos conceitos da 14.0 associando ao território brasileiro obteve-se que a literatura sobre o assunto ainda é escassa, sendo que a proposta afeta essencialmente a fabricação, para a customização do produto final, com investimentos em equipamentos, gestão e segurança de dados, e formação de novas habilidades dos trabalhadores (FREITAS, 2016).

Cardoso (2017) discute que existe uma distância para que o Brasil atinja patamares similares aos países desenvolvidos, já que a formação universitária e de técnicos está distante da realidade empresarial, os investimentos em tecnologias de ponta e, que devem ser apoiados por políticas governamentais direcionadas para o desenvolvimento.

No artigo de Rita et al (2015), coloca que os investimentos no setor industrial incluem pesquisas, universidades, formação profissional e no sistema de ensino, coordenação política em diferentes órgãos ministeriais e diferentes níveis e financiamento responsabilizado pelo nível federal e estadual. A autora ainda considera que o sistema brasileiro de inovação é composto essencialmente por instituições públicas, como a Embraer, Petrobrás e Embrapa, sendo que:

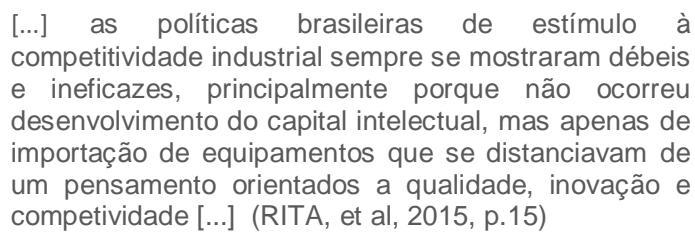

No setor da construção civil e arquitetura não é diferente, como previsto nos textos, a temática Indústria 4.0 ainda está distante dos processos construtivos e da gestão do empreendimento. São presentes algumas discussões a respeito de sistemas de controle de veículos leves sobre trilhos e controle de sistemas de abastecimento de água. 
O setor dos empreendimentos da construção civil têm que ser analisados com a devida especificidade, dado que o seu produto está inserido em uma cadeia específica, e que os conceitos da 14.0 se inserem no âmbito da comercialização, da produção, da gestão ou da elaboração do seu projeto. Como refere Miyasaka, Paoletti \& Fabrício (2016) o processo é auxiliado pelas tecnologias, no armazenamento de dados, na gestão do projeto e do empreendimento através dos programas BIM e dos equipamentos digitalizados que contribuem para a produção do edifício.

Sendo assim, o setor da construção civil e da arquitetura, também se encontram distantes de atingir as formas organizacionais da Indústria 4.0, visto que se trata de um ambiente onde as diretrizes políticas não estão voltadas para os investimentos do setor assim como os setores da indústria propriamente dita.

Ao mesmo tempo, as pesquisas demonstram que os pesquisadores estão se apropriando dos conceitos e teorias que tratam da proposta, e aos poucos experimentam e refletem sobre a temática adequando as proposta ao contexto no território brasileiro.

\section{DISCUSSÃO}

Para que nosso país atinja patamares de produção da Indústria 4.0 são necessários vários esforços que de antemão não constituem interesse nos países emergentes. Realizar a pesquisa exploratória e identificar os níveis de discussão a respeito do assunto contribui para que a industrialização brasileira seja adequada à realidade local em futuras perspectivas.

Para atingir plenamente o contexto da 14.0 no setor da construção civil, há a necessidade de se enfrentarem os desafios com plataformas independentes de fabricação, aumento dos níveis de automação, melhorias nos equipamentos de produção para benefício dos ambientes de chão de fábrica, componentes com melhores eficiências energéticas e implantação de fibras óticas para melhoria da capacidade de comunicação interna e externa da manufatura (LEONARD, 2015).

As empresas têm que lidar com mudanças organizacionais e de processos, com elevados custos de implementação e a previsão pouco clara de redução de investimentos ou com a crescente necessidade de segurança e proteção de dados. Do ponto de vista técnico, existem vários problemas a serem enfrentados, como a falta de padrões para tecnologias, os requisitos para equipamentos ou necessidades crescente de redes de comunicações avançadas, incertezas jurídicas e contratuais (BRYNJOLFSSON; McAFEE, 2014).

A automação interferindo no projeto considera as diversas etapas, tais como a integração, organização, aspectos informais e tecnologia das máquinas, que melhoram a eficiência, qualidade e complexidade do produto. São verificados também a logística, a montagem, o grau de estruturação de automação na fábrica e no local, o desenvolvimento a partir da coordenação modular, o controle de variações e a relação da fabricação com as necessidades do cliente.

Os processos de automatização e suas aplicações na construção civil, como descrevem Balaguer; Abderrahim (2008), podem ser divididos em dois grandes grupos: o primeiro caracteriza-se pela utilização de robôs em ambientes externos para a área de infraestrutura, como pavimentação e compactação de estradas, túneis e construção de pontes, escavações, dragas, inspeção e manutenção de setores urbanos etc. O segundo grupo está na área de construção de moradias, na construção e montagem de estruturas, montagem de painéis de vedação, compactação de concreto, acabamentos finais internos ou montagem do edifício completo.

A maior dificuldade da Robótica e Automação em Construção (RAC) está relacionada com o ambiente de trabalho, que, em geral, é pouco estruturado. Trabalhar nesses locais envolve objetos pesados, elementos com grande tolerância, baixos níveis de padronizações, insuficientes níveis de industrialização e pré-fabricação, além do número de atores. Há que se fazer um esforço para aumentar o nível de automação envolvendo processos nesse importante setor econômico com a finalidade de incrementar sua produtividade (BALAGUER; ABDERRAHIM, 2008).

Além disso Oesterreich; Teuteberg (2016) referem que os aspectos importantes para a implementação da automatização na área da construção civil são:

1. Mudança de atitude nas empresas, nas máquinas da indústria, nos centros de pesquisa e nos direcionamentos governamentais, no sentido de se desenvolverem novos produtos comerciais com tecnologias de ponta;

2. Implementação de novas tecnologias de informação, mudando os processos de trabalho em todos os segmentos,

3. O mercado da globalização e, consequentemente, a adaptação da estrutura comercial no setor da construção civil introduzem um alto nível de competitividade, o que força as empresas a adotarem técnicas automatizadas e mais eficientes.

As tecnologias avançadas de pesquisa têm utilizado vários meios para a elaboração de operações computadorizadas na construção civil. Esses equipamentos robóticos podem ser altamente flexíveis, uma vez que podem trocar a função a partir da troca da ponta que define diferentes ações, como processos aditivos com argamassa, subtrativos como fresadoras, transformação, com modificação de materiais e montagem, com posicionamento de componentes.

Em casos recentes, os autores referem que as políticas de planejamento que utilizam o conceito das smart cities estão voltadas para o emprego da tecnologia na integração para a educação, na comunicação entre comunidades, nos sistemas de infraestrutura, no acesso amplo à internet, na gestão de uma rede de cidades, na mobilidade controlada e informada, no monitoramento climático, dentre outros projetos, integrando uma diversidade de informações para a maior previsibilidade e busca de soluções de problemas de forma antecipada, que resultem em uma qualidade de vida superior dos moradores e que atente para a manutenção do meio ambiente (MONZON, 2015).

O cenário é otimista, com uma crescente aplicação de tecnologias inovativas em nosso país. Claro que não há a ansiedade de aplicação aos moldes dos processos industrializados dos países industrializados, mas sim encontrar os meios e melhores utilizações para a nova forma de organização da 14.0 que certamente traz benefícios para todos os envolvidos no processo. 


\section{AGRADECIMENTOS}

Agradecemos aos Grupos de Pesquisa ActLab e ArquiTec, à Coordenação de Aperfeiçoamento de Pessoal de Nível Superior - CAPES _ PDSE processo no. 99999.003519/2015-05 o apoio para divulgação do trabalho do Programa de Pós-Graduação e do Departamento de Arquitetura e Urbanismo da Universidade de Viçosa - MG (UFV). Escritório Rimond de Gestão e Desenvolvimento de Projetos, Eng Giuseppe Chiarandá, Escritório Ramboll de Gestão e Desenvolvimento de Projetos, Também aos pesquisadores Roberto Naboni, Maya Zheilazkova, Arq. Ommer Mert Cek, Eng. Roberto Ferrari, Eng. Gary Tortona, Arq. Francesco Catalano e Arq. Bernardo Jacobsen.

\section{REFERÊNCIAS}

ABRAMOVICI, M.; GÖBEL, J. C.; NEGES, M. (2015). Smart Engineering as Enabler for the 4th Industrial Revolution, In: FATHI, M. (Ed.) Integrated Systems: Innovations and Applications, Siegen: Springer. p. 173-194.

ANTONIO, D. (2016). Personalizzazione di Massa - La Diffusione dell'Internet delle cose Permetterà alla Manifattura di Accendere un Dialogo Continuo tra Prodotto, Machina che lo Fabbrica e Consumatore Finale. Milano: Nòva Edu - Lezione di Futuro - 07, II suo 24 ore.

ASSAD NETO, A. et al. (2015). A busca de uma identidade para a indústria 4.0, Brazilian Journal of Development, v.4, n.3, p. $715-732$.

BALAGUER, C.; ABDERRAHIM, M.(2008). Trends in Robotics and Automation in Construction, p.1-22. In: BALAGUER, C.; ABDERRAHIM, M.(2008) Robotics and Automation in Construction, Europe Union: InTech.

BRETON, P.; PROUX, S. (2006). Sociologia da Comunicação Tradução: Ana Paula Castellani, 2a. ed., São Paulo: Loyola.

BRYNJOLFSSON, E.; McAFEE, A. (2011). Race Against The Machine: How the Digital Revolution is Accelerating Innovation, Driving Productivity, and Irreversibly Transforming Employment and the Economy. Massachusets: Digital Frontiers Press.

CARDOSO, W. (2017). Digital Manufacturing, Industry 4.0, Cloud Computing and Thing Internet: Brazilian Contextualization and Reality, Independent Journal of Management \& Production (ljm\&P), v. 8, n. 2, p. 459 - 474.

CERQUEIRA, V. (2016). Tecnologia em Design de Vidros Conformados: uma proposta para ganhos em competitividade na Indústria 4.0, Blucher Design Proceedings, Congresso Brasileiro de Pesquisa e Desenvolvimento em Design, v.1, n.1, p. 4539 - 4552.

CLAUDIA, Ana et al.. (2017). A MODULARIZAÇÃO E A INDÚSTRIA 4.0.. In: Anais do II SIGEPRO - Simpósio Gaúcho de Engenharia de Produção. Anais...Novo Hamburgo(RS) Feevale. Disponível em $<$ https//www.even3.com.br/anais/sigepro2017/51301-AMODULARIZACAO-E-A-INDUSTRIA-40>. Acesso em: 01/07/2018 12:43

D'AGostINI, M. A. da S.; SOBRINHO, S. R. L. (2016). Industria 4.0 - Futuro Promissor Para a Modalidade De Transporte VIt (Veículo Leve Sobre Trilhos) Industry 4.0 -Promoting Future for Vlt (Light Vehicle on Tracks), Ignis, Caçador, v.5, n.2, p. $26-32$.

FREITAS, M. M. B. C. de; FRAGA, M. A. de F.; SOUZA, G. P. L. de (2016). Logística 4.0: conceitos e aplicabilidade: uma pesquisa-ação em uma empresa de tecnologia para o mercado automobilístico, Núcleo de Pesquisa Acadêmica: PAIC.
KATSUNDO, H. (1994). Automation - its concept and a short history. Technovation, v.14, n. 2, p.121-128.

LARIZZA, A. (2016). La Rinascita della Fabrica: Nell'era della Manifattura Digitalizzata, Le Aziende Possono Tornare a Creare Valore a una Condizione: L'uomo dourà Competere al Fianco delle Machine In: La Fabrica Digitale: Come Funziona L'Industria 4.0 e che cosa Possiamo Fare. Milano: Nòva Edu - lezione di Futuro - 07, II suo 24 ore.

LASI, H. L.; KEMPER, H.-G.; FETTKE, P.; FELD, T.; HOFFMAN, M. (2014). Industry 4.0. Business \& Information Systems Engineering, v.6,n.4, p.239-242.

LEE, J.; KAO, H.-A.; YANG, S. (2014). Service innovation and smart analytics for Industry 4.0 and big data environment. Procedia CIRP, v.16, p. 3-8.

LEONARD, J. (2015). Building Tomorrow's Delivering the benefits of Industry 4.0 requires robust communication. Professional Engineering, v.28, n.2, p.16.

MATTELART, A. (2002). História da Sociedade da Informação. Tradução: Nicolás. Nuimi Campanário, São Paulo: Editora Loyola.

MIYASAKA, E. L.; PAOLETTI, I.; FABRÍCIO, M. M. (2016). Choosing the Partner in a Digital Fabrication Case, Blucher Design Proceedings... XX Congress of the Iberoamerican Society of Digital Graphics, v. 3, n. 1, p. $374-380$.

MONZON, A. (2015). Smart Cities Concept and Challenges: Bases for the Assessment of Smart City Projects, in: $M$. Helfert et al. (Eds.): Smartgreens 2015 and Vehits 2015, CCIS 579, pp. 17-31. DOI: 10.1007/978-3-319-27753-0_2

NABONI, R.; PAOLETTI, I. (2015). Advanced Customization in Architectural Design and Construction. Milano: Politecnico di Milano - Springer.

OESTERREICH, T. D.; TEUTEBERG, F. (2016). Understanding the implications of digitization and automation in the context of Industry 4.0: A triangulation approach and elements of a research agenda for the construction industry. Computers in Industry, v.83, p.121-139.

POSADA, J.; TORO, C.; BARANDIARAN, I.; OYARZUN, D. (2015). Visual Computing as Key Enabling Technology for Industrie 4.0 and Industrial Internet. IEEE Computer Society, v.26, p. 1-11.

RITA, L. P. S. et al (2016). Sistema Nacional de Inovação: uma análise dos sistemas na Alemanha e no Brasil, Navus Revista de Gestão e Tecnologia, v. 6, n. 4, p. 06-25.

SHAFIQA, S. I.; SANINA, C.; SZCZERBICKIB, E.; TOROC, C. (2015). Virtual Engineering Object/virtual Engineering Process: A specialized form of Cyber Physical System for Industrie 4.0. Procedia Computer Science, v. 60, n.1, p. 1146-1155.

SILVEIRA, L.; LIMA, W. Q. (2003). Um breve histórico conceitual da Automação Industrial e Redes para Automação Industrial. Lagoa Nova: UFRN-PPgEE.

SOMMER, L. (2015). Industrial revolution - industry 4.0: Are German manufacturing SMEs the First Victims of this revolution? Journal of Industrial Engineering and Management, v. 8, n.5, p.1512-1532.

TRENTESAUX, D.; BORANGIU, T.; THOMAS, A. (2016). Emerging ICT concepts for smart, safe and sustainable industrial systems. Computers in Industry, v.6, n.4, p. 1-10.

WANG, S.; WAN, J.; ZHANG, D.; LI, D.; ZHANG, C. (2015). Towards smart factory for Industry 4.0: A self-organized multi-agent system with big data based feedback and coordination. Computer Networks, v.101, p. 158-168. 